\title{
Sachregister zu Band 56
}

Die fett gedruckten Zahlen bezeichnen Eigenarbeiten. Bb. = Buchbesprechung. Ablatio retinae, Entstehung der $-\cdot 243$.

- $\quad$ operativ geheilte -. 196.Abduzenslähmung, rezidivierende

- im Zusammenhang mit

Schwangerschaft. 267. Aderhaut s. Chorioidea. Adrenalin, Einfluß des - auf den Augendruck bei Glaukom. 268.

- $\quad$ Wirkung des - bei Glaukom.189.

Adrenalinmydriasis und Insu-

linmiosis. 215. Akkomodationsmechanismus,

Wahrscheinlichkeitsbeweis für den

Helmholtzschen -. 127. Altersveränderungen, physiolo-

gisch-chemische - des Auges.

127. Amotio retinae acquisita bei

Vater und Sohn. 155. Anatomie des Auges. 124ft. Antitonen. 145. Argentum nitricum, Hornhaut-

verätzungen durch -. 242. Argyrol. 132. Arteria centralis retinae, Be-

stirnmung des Blutdruckes in der

-. 130. Astigmatism us in versus corneae

bei Myopen und im Senium. 320. Aubert-Förstersche Erschei-

nung. 260. Augenerkrankungen im Kindes-

alter. 74 (Bb.). Augenheilkunde. 74 (Bb.), 272

(Bb.), 274 (Bb.). Augenhintergrund, Aufnahme

des -. 200.

- $\quad$ naevoide Degeneration des -.139, 256.

Augenlider s. Lider. Augenmuskellähmung, angebo-

rene. 361. Augenmuskelwirkung, Schema

der -. 64. Autophthalmoskopie. 61. Axonoskop. 258.

B.

Bäderbehandlung bei Augen-krankheiten. 249.

Bakterienwachstumim Kammer-wasser, Einfluß des Glaskörpers auf das -. 271.

Bellsches Phänomen. 233.

Bergleute, Das Wundgeschwür der

- 1.359-Berufskrankheiten, Ausdehnung

der Unfallversicherung auf -.

150. Bestrahlungstherapie bei Ulcus

corneae. 231. Betriebsunfälle, Gleichstellung

der Gewerbekrankheiten mit den

-. 348. Bewegungstäuschung, optische 
-. 146. Bindehaut s. Conjunctiva. Binokularsehen und Sehnerven-

kreuzung. 346. Blindheit, Ätiologie der jugend-

lichen --. 264. Blut, Abhängigkeit des intraoku-

laren Druckes von der aktuellen

Reaktion des -. 185, 239. Blutdruck in der Arteria centralis

retinae. 130. Blutserum, Beziehungen zwischen - und intraokularer Flüssigkeit.

127. Blutungen, Beziehungen zwischen

subarachnoidalen und intraoku-

laren -. 69. Brillen. 62. Brillengläser, Bestimmung der

Brennweite von -. 62. Bulbusdislokation, hochgradige

- bei Depressionsfraktur des

Jochbeins. 333. Bulbusoperationen, schwierige

-. 164.

C. Canalis hyaloideus, Untersuchun-

gen über die Persistenz des - -.

126. Chalazion, Pathologic des -. 218,

244. Chiasma nervorum opticorum

und Binokularsehen. 346. Chininamaurose. 267. Chlamydothrix ochracea-In-

fektionen, kulturell erwiesene

Fälle von -. 259. Chorioidea, Blutzirkulation in den

Gefäßen der -. 130.

380

Sachregister.

Chorioidea, Hereditäres Kolobom der-. 363.

Multiple Tuberkel in der -. 264.

Operation eines Leukosarkoms der

- $\quad$ mit Erhaltung des Auges. 252.Chrysarobinschädigung der

Hornhaut. 140. Colo bom, hereditäres - der Iris

und Chorioidea. 363. Coloboma palpebrae congeni-

tum. 339. Conjunctiva als Eintrittspforte in-

íektiöser Prozesse der Orbita. 197.

- Pemphigus der -. 139.

- $\quad$ Sarkom der-. 106.Conjunctivitis mit Erkrankung

der Tränendrüsen. 370.

- Neuropathische. 148.Cornea, Chrysarobinschädigung der

-140 .

- $\quad$ Dystrophia epithelialis der -.242 .

-- Epithelfasersystem der -. 125, 241.

Hämatom der -. 72.

Pigmentierungen und Pigment-ringe am Rande der -. 125. -trübung, gleichartige angeborene

bei Zwillingen. 361 .

-trübung, ringförmige epitheliale nach Tonometrie. 235.

Veränderungen an der Hinter-fläche der -. 241.

Verätzungen der - durch Per-chlorsäure und Argentum nitri-cum. 242.

- $\quad$ Wirkung kurzwelligen Lichtes aufdie Regeneration der -. 230. 
Corpus vitreum, Absaugung des

- $\quad$ bei Retinitis proliferans. 370 .

Beobachtungen am -. 125.

Eigenschaften des des Tier-

auges. 127.

- $\quad$ Einfluß des - auf das Bakterien-wachstum im Kamme $\pi v a s s e r .271$.

- $\quad$ Rezidivierende Blutung des -.261.

- $\quad$ Zystizerkus im -. 143.

I).

Dakryolith im unteren Tränenröhr-chen. 264.

Descemeti-Ablösung nach Star-operation. 137.

Deviation der Augen nach oben, periodische assoziierte - bei Encephalitis epidemica. 237.

Diabetes mellitus, Augendruck bei -. 144, 231.

Doppelbilder, Untersuchung auf

- $\quad$ bei Farbenblindheit. 57.Doppeltsehen, Instrumentarium

zur Untersuchung des -. 64.

Druck, intraokularer, Abhängigkeit des - von der aktuellen Reak-tion des Blutes. 185, 239.

- Abhängigkeit des - von cler W asserstof f ionenkonzentration des Kammerwassers. 128.

-— bei Zuckerkranken. 144, 231.

- Einfluß des Adrenalins auf den - bei Glaukom. 268.

- $\quad$ - Einfluß der Radiumemanationauf den -. 140.

- $\quad$ Erhöhung des - bei einemSympathikotoniker. 68.

- $\quad$ - Gleichzeitige Registrierungdes Lumbaldruckes und des -.

$\mathrm{i} 45$.

- $\quad$ - Herabsetzung des - durchAntitonen. 145.

- $\quad$ - Mechanik der Schwankungendes - beim primären Glaukom.183.

- $\quad$ - Schwankungen des - am ge-sunden und glaukomatösen Auge.

372-Druckverband, Wirkung des -.

225. Dystrophia adiposo-genitalis

mit Augenstörungen. 364. Dystrophiaepithelialiscorneae

242.

E.

Echinococcus orbitae, entfernt durch Orbitotomia superciliaris.

67. Einschlußerkrankungen, Ein-

teilung der -. 109. Ektropium der Lider nach Karies

des Orbitalrandes. 367.

- uveae. 267.

Ekthyma terebrans, als Ursache von Lidgangrän und Keratoma-lazie. 267.

Elektroretinogramm. 130, 131.

Endokrine Störung als Ursache der Keratitis. 235.

Endophthalmitis phakoana-phylactica. 261.

Entropiumoperation. 140.

Enzephalitis epidemica, ein be-sonderer Tic bei -. 237.

Epithelfasersystem der Hornhaut. 125, 241.

Erweichungsprinzip bei der Glaukombehandlung. 189.

Erythema exsudativum multi-forme. 139. 
Exophthalmus bei Allgemeiner-krankungen. 244.

F.

Farbenblindheit, die verschiede-nen Typen der -. 73 (Bb.).

Sachregister.

381

Farbenblindheit, Untersuchung $\mid$ auf Doppelbilder bei -. 57 .

Farbensinn der Bienen und der Fische. 73 (Bb.).

- Untersuchung des. 59.Farbensysteme, anomal-trichro- j

matische. 206. Fazialisschädigung, ungewöhn- !

liche Augenstörungen nach -.

150. Fixationspinzette, dreifach ge-

gabelte. 199. Flüssigkeitswechsel, intraoku-

larer -. 128, 129, 130. Foramen opticum, Y¹/8rgrößerung

des -. 211. Fremdkörper, postäquatoriell ein-

gedrungener - im hinteren Bul-

busabschnitt. 44. Frühj ahrskatarrh, allergische

Reaktion bei -. 71 .

- $\quad$ Zur Histologie des -, 21.Führungsbewegungen. 209.

G.

Ganglion Gasseri, Ursache der

Keratitis nach Operationen am

---. 270. Gesichtsfeldaufnahmen, Grund-

lagen zur Vereinheitlichung der

-· 205. Gewebsatmung im Auge. 182. Gewerbekrankheiten, Gleich-

stellung der - mit den Betriebs-

unfällen. 348. Gewerbliche Verletzungen des

Auges. 151 (Bb.). Glaukom, Adrenalinwirkung bei -.

189.

Einfluß des Adrenalins auf den

Augendruck bei -. 268.

- $\quad$ Erweichungsprinzip bei der Be-handlung des -. 189.

- $\quad$ Mechanik der Augendruckschwan-kungen beim primären -. 183.

- $\quad$ Schwankungen des intraokularenDruckes bei -. 372.

- $\quad$ Sekundäres - durch Kataraktatumescens. 263.

Glaukomiridektomie, Dauerer-

gebnisse nach -. 143. Glaukomoperationen, vorberei-

tende Maßnahmen von -. 40. Glioma retinae, Anatomie des

181. Gonioskopie. 149.

H.

Haenelsches Symptom, Messung und klinische Bedeutung des -. 219.

Hämatom der Cornea. 72.

Hemeralopie mit Myopie, Ver-

erbung der -. 374.

Hemianopsie, homonyme - bei

hochsitzender Unterbrechung der 
optischen Bahn. 146.

Hemiatrophia facialis progres-

siva mit Augenhintergrundsver-

änderungen. 254, 367. Heringsches Gesetz, Beweise für

das - aus dem Nystagmus der

Bergleute. 132. Herpes corneae, Rezidiv bei-.

212. Herpesvirus, Bedeutung des für

die Ophthalmia sympathica. 213. Heterochromie, Sympathikus-

lähmung als Ätiologie der -. 207. Höhenschielen, scheinbares. 247. Holokaintrübung der

Hornhaut.

235-Hordeolosis, Thrombophlebitis or-

bitae und Sinusthrombose nach-.

248. Hydrophthalmus congenitus

bei einer Katze. 251. Hypophyse und Auge. 131. Hypophysistumor, Fälle von ope-

riertem -. 136 .

I.

Immunität, örtliche. 226.

Insulin, Einhalten der Gangrän eines Bindehautlappens durch Be-handlung mit -. 369.

Insulinmiosis und Adrenalin-mydriasis. 215.

Intraokulare Flüssigkeit, Be-ziehungen zwischen Blutserum und -. 127.

Intraokularer Druck s. üruck.

Intraokularer Flüssigkeitswechsel. 128, 129, 130.

Iridozyklitis, Ätiologie der -. 138.

- und Zähne. 138.

Iris, Melanosarkom der -. 197.

Iriseinklemmung, Behandlung der -. 240.

Iris-Kammerwinkel, Untersuchung des -. 149.

Iriskolobom, hereditäres - mit eigenartiger Irisanomalie. 363.

Iriszyste, trepanierte. 265.

Irradiation und Simultankontrast. 144.

J.

j o c h b e i n, mit hochgradiger Bulbus-dislokation komplizierte, opera-tiv geheilte

Depressionsfraktur des -. 333.

J odnatriumbehandlung, intra-venöse. 374 .

382

Sachregister.

K.

Kainon. 266.

Kammerwasser, Abhängigkeit des

intraokularen Druckes von der

Wasserstoffionenkonzentration

des -. 128. •- Einfluß des Glaskörpers auf das

Bakterienwachstum im -. 271.

- $\quad$ Messung der Wasserstoffionenkonzentration im - mit Hilfe derGaskette. 200.

- $\quad$ Untersuchung des -. 127, 128.Karzinommetastase. 363.Katarakt, experimentelles - in- 
folge von Nährschaden. 198. Katarakta diabetica, Ätiologie der -. 232. Katarakta senilis, eine neue

Theorie der -. 141.

- Gibt es einen Antagonismus zwi-schen - - und Haabscher se-niler Makulaveränderung ? 79.

Katarakta tumescens, Glaukom, verursacht durch -. 263.

Kataraktoperation bei Mikro-phthalmus. 365.

Descemeti-Ablösung nach-.137.

Fistulierung durch Einklemmung der sichtbaren Linsenkapsel in die Wundränder nach -. 255.

Verbesserungen in der Technik der -. 132.

Vermeidung der letzten Wundin-fektion und Komplikationen bei der -. 220.

Keratitis auf Grund endokriner Störung. 235.

Ursache der - nach Operationen am Ganglion Gasseri. 270.

parenchymatosa, Kombina-tion von antiluetischen und fieber-erregenden Mitteln bei - . 262.

- $\quad$ rodens. 141.Keratomalazie, verursacht durch

Ekthyma terebrans. 267. Klimatische Einflüsse bei Au-

genkrankheiten. 249. Knochenbrüchigkeit, gemein-

sames Vorkommen von blauen

Skleren mit - und Schwerhörig-

keit. 150. Koch-Weeks-Problem. 226. Kokainersatzmittel. 170.

L.

Lagophthalmusparalyticus, Re-sektion des Orbicularis und beider Lidbänder bei -. 117.

Leukosarkom der Chorioidea, Operation eines - mit Erhaltung des Auges. 252.

Lichen conjunctivae. 228.

Licht, anormale und krankhafte

Wirkungen des - auf das Auge.

151 (Bb.). -- Wirkung kurzwelligen - auf die

Regeneration der Hornhaut. 230. Lidbänder, Resektion beider -

bei Lagophthalmus paralyticus.

117. Lider, Immobilisation der - bei

bulbuseröffnenden Operationen.

263. Lidgangrän, verursacht durch Ek-

tyma terebrans. 267. Lidsperrer. 263. Limbus corneae, Lymphgefäß-

system am -. 125. Lipoidstoffwechsel des Auges.

237-Lues, Bedeutung der -- íürAmotiv

und Glaukom. 244. Lumbaldruck, gleichzeitige Re-

gistrierung des Augendruckes und

des -. 145. Lupus des Auges, behandelt mit

Pyrogallussalbe. 369. Lymphgefäßsystem am Limbus

corneae. 125.

M.

Macula, Gibt es einen Antagonismus zwischen Cataracta senilis und Haabscher seniler Veränderung der -? 7Í).

Makuladegeneration, scheiben-förmige. 194.

Magnet, Zusammenarbeiteñ des --mit der Röntgenphotographie.

$195-$ 
Melanosarkom der Iris. 197.

M i k r o p h t h a $1 \mathrm{~m} \mathrm{u} \mathrm{s,} \mathrm{Kataraktoperation} \mathrm{bei} \mathrm{-.} 365$.

Mikroskopie des lebenden Auges. 56. $57>$ I25 > 126, 152 (Bb.).

Mikuliczsche Krankheit mit Au-gensymptomen. 221.

Mitbewegungen, pathologische -der Pupille. 140.

Myopiegenese, zur Kritik der 351, 356.

N. Naevoide Pigmentierung des

Fundes 139, 256. Nährschaden, experimenteller

Star infolge von -. 198. Nebennierenhormon, antagoni-

stische Wirkung von Pankreas-

hormon und -. 215. Nervus opticus, Anatomie des - .

126.

atrophie, Therapie der --

bei Tabes. 245. - - bei Tabes und Paralyse. 180.

Sachregister.

383

Xervus opticus, Erkrankung des -. 246.

- Fortleitung der sympathisie-renden Entzündung in den -. 1275.

- Spat aufgetretene Erkrankung des - nach stumpier Schädelver-letzung. 38.

- - Sympathische Entzündung

des -. 196. Netzhaut s. Retina. Neubildung, wurmfortsatzähn-

liche. 240. Normalperimeter. 205. Nystagmographie. 57, 65. Nystagmus, Apparate zur Unter- j

suchung des -. 64.

der Bergleute, Beweise für das Heringsche Gesetz aus dem -. 132.

Fernübertragung des - auf elek- i trisch-optischem Wege. 371.

Willkürlicher. 143.

0 .

Oguchische Krankheit, Sektion

eines Falles von. 131. Ophthalmia lenta. 146. Ophthalmia sympathica. 196, 213.

- $\quad$ Fortleitung der - in den Seh-nerven. 275.

Ophthalmic Year Book. 378

(Bb.). Optische Bahn, vollständige ho-

monyme Hemianopsie bei hoch-

sitzender Unterbrechung der -.

146. Optisch-motorische Erlebnis-

e in h e it, das physiologische Sub-

strat der -. 211. Orbicularis, Resektion des - bei

Lagophthalmus paralyticus. 117. Orbitalerkrankungen, akut ent-

zündliche. 302. Orbitalgeschwülste. 248. Orbitalphlemone, doppelseitige

chronische. 221. Orbitalrand, Ektropium der Lider

nach Karies des -. 367. Orbitotomia superciliaris zur

Entfernung eines Echinococcus

orbitae. 67.

P. 
Pankreashormon, antagonistische

Wirkung von Nebennierenhormon

und -. 215. Parallaxenrefraktometer, Ver-

besserungen am -. 60. Paralyse, Optikusprozeß bei -.

180.

Parinaudsche Conjunctivitis.

68. Pemphigus der Conjunctiva. 139. Perchlorsäure, Hornhautver-

ätzungen durch -. 242. Perimetrie. 58, 59, 205. Photoelektrische Reaktion des

Froschauges. 131. Photographie des lebenden Tier-

auges. 241.

- des vorderen Augenabschnittes, Stereokamera zur -. 55, 56.

Physiologie des Auges. i26ff. Pigmentierung, naevoide - des

Fundus. 139, 256. -: und Pigmentringe am Hornhaut-

rande. 125. Pilzerkrankungen des Auges. 234. Pilzkonkrement im oberen Trä-

nenröhrchen. 264. Plastik der Übergangsfalte. 366. Plastische Operationen. 199.

Prismenrefraktometer 60, 257. Pseudogliom. 244. Pseudogliomacongenitum, fami-

liäres Auftreten von -. 155. Psicain. 68, 262. P u p i 11 e, pathologische Mitbewegun-

gen der $-^{\wedge}-$. 140. Pupillenbewegung. 361. Pupillenstörungen, akut auftre-

tende bzw. transitorische. 217. Pyrogallussalbe zur Behandlung

des Augenlupus. 369.

R.

Raddrehung des Auges. 145. Radiumemanation, Einfluß der

- auf den intraokularen Druck.

140. Rectus superior, isolierte Ab-

lösung des - nach stumpfem

Trauma. 174. Reizkörpertherapie. 262. Refraktion, Bestimmung der--.

$60 \mathrm{ff}$. Retina, arterielle und venose Zir-

kulationsstörungen der -. 194

Doppelte Gefäßversorgung der -. 199.

Erkrankung der -. 246.

Faserverlauf in der -. 126.

Pigmentverschiebung im Epithel der -. 131.

Stoffwechselvorgänge in der -. 202.

Theorie des Blutumlaufs in der

- i93. Retinalvenenpuls, Entstehung

und Bedeutung des -. 192. Retinaödem, Befund bei einem

Fall von -. 140. Retinitis albuminurica. 144.

384

Sachregister.

Retinitis proliferans, Glaskör-perabsaugung bei -. 370 .

Röntgenbehandlung. 265.

Röntgenlokalisation. 195.

RöntgenologischeFortschritte. 375-

S. 
Sanochrysin, bei experimenteller

Augentuberkulose. 223. Sarkom der Bindehaut. 106. Schädelgehirnschuß, Spätfolge

nach -. 150. Schädelverletzung, spat aufge-

tretene Sehnervenerkrankung

nach stumpier -. 38. Schalenprothesen mit Löchern

zum Fixieren. 366. Scheitelbrechwertmesser. 62,

144. Schrotkornverletzung. 144. Schwerhörigkeit, gemeinsames

Vorkommen von blauen Skleren

mit Knochenbrüchigkeit und -.

150. Schußanalyse, Grundsätze für die

-. 151. Sehnerv s. Nervus opticus. Sehproben. 62, 63. Sehsphäre, Stoffwechselvorgänge

in der - . 202. Sella turcica, Röntgenologie der

-. 144. Sémiologie oculaire. 74 (Bb.). Sepsis orbitae lenta. 221. Sideroskopie. 60.

Simultankontrast und Irradiation

144. Sinusthrombose nach Hordeolo-

sis. 248. Skiaskopie. 61. Skiaskopiermodell. 253. Skier a, gemeinsames Vorkommen

von blauen - mit Knochenbrüchigkeit und Schwerhörigkeit.

150 .

multiple Tuberkel in der -. 264. Skotom, kann - -bildung allein

durch Erhöhung des intraokularen

Druckes bedingt sein ? 48. Spaltlampe. 56, 57, 125, 126, 152

(Bb.). Stäbchenapparat, Funktion des

- 73 (Bb.). Stereokamera zur Photographie

des vorderrn Augenabschnittes.

55-Sympathikotonie, Druckerhö-

hung bei. 68. Sympathikuslähmung alsAtiolo-

gie der Heterochromie. 207. Syphilis s. Lues.

$\mathrm{T}$.

Tabes, Optikusprozeß bei -. 180.

- $\quad$ Therapie der Optikusatrophie bei-· 245.

Tebeprotin Toeniessen 222, 224. Thermokauter, Behandlung mit

dem - von Wessely. í)0. Thrombophlebitis orbitae nach

Hordeolosis. 248. Tonometer von Wendt. 141. Tonometeraichung. 54, 55. Tonometrie,

ringíörmige epithe-

liale Hornhauttrübung nach -.

235. Tonusänderungen auf optische

Reize. 200. Trachom. 10Ö, 224, 376 (Bb.). Tränendrüsen, Conjunctivitis mit

Erkrankung der -. 370. Tränenorgane, Pathologie der - .

152 (Bb.). Tränenpunktmangel, angebore-

ner. 68. Tränenröhrchen, Pilzkonkrement

im oberen -. 264.

- $\quad$ Überzähliges 68.Tränensack, chirurgische Behandlung der Tuberkulose des -. 266.

Tränensackeiterung. 248. Tränenträuf eln. 240. Tränenwege, Wiederherstellung

der - durch Fortpflanzung von

Haut-Epidermislappen. 373. Tuberkel, multiple - in der Cho-

rioidea und Sklera. 264. Tuberkulose des Auges, trachom- 
ähnliche. 261.

- des Tränensacks, chirurgische Behandlung des -. 266.

der Uvea. 265. - Sanochrysin bei experimenteller

- des Auges. 223. Tunica vasculosa lentis, persi-

stierende. 244. Tutokain. 262.

v.

Ulcus corneae, Bestrahlungsthera-pie bei -. 231.

Ultraviolettbehandlung des Auges. 246.

Unfallkunde. 150, 375.

Untersuchungsmethoden. 53ff., 273 (Bb.).

V.

Venenpuls. 130. Verätzung des Auges. 265. Verletz ungen, gewerbhche - des

Auges. 151 (Bb.). Versicherungskunde. 150, 375. Vitiligo. 35. Vorlagerung, Technik der - . 250.

Sachregister. 385

W.

Wasserstoffionenkonzentra-tion des Kammerwassers, Ab-hängigkeit des intraokularen Druckes von der -. 128.

Wasserstoffionenkonzentra-tion im Kammerwasser, Messung der - init Hilfe der Gaskette. 200.

Wörterbuch, fremdsprachliches optisches. 152 (Bb.).

Wundgeschwür der Bergleute. 1, 359-

Z.

Zähne und Iridozyklitis. 138. Zentralgefäßstämme, Inhalt der

- bei Leichenaugen. 198. Ziliarepithel. 196. Ziliarnervenschleifen, intra-

sklerale. 197. Zonula Zinnii. 125. Zyklodialyse. 266. Zvstizerkus im Glaskörper. 143.

Namenregister zu Banð 56.

Die fettgedru

A.

Abarbanell 150. Albrich 234. Altland 360. Arnold 54, 55. Ascher 57, 125, 141, 142, 143, 186,

216 ,

$231-$,

Aszatós 67. Avizonis 339.

Axenfeld 197, 220.

B.

Bachstez 59, 174. Back 138. Bar 360.

Bart els 65, 66, 191, 210, 222, 233, 346, 360, $361>365.367,369,37^{\circ}$.

Bäumler 62.

Baurmann 127, 186, 192, 200.

Behr 254.

Beselin 246, 247

Bietti 272.

Birch-Hirschíeldl( > 4, 224.

Birkhäuser 63.

Birnbacher 124.

Bistis 105, 207.

v. Blaskovics 68, 264, 265, 266.

Blenke 150. 
Bliedung 130.

Bottrop 1.

Brana 68.

Brons 361.

Bruckner 205, 207.

C. Comberg 54, 58, 109, 125, 130, 195, 205, 225, 234.

ckten Zahlen bedeuten Ei

Con in 196. Cords 194, 109. Cronstedt 271. v. C s a p o d y - M ó c s y

265. Cuperus 144.

D.

Dieter 78 (P.).

v. Ditrói 259, 264, 266,

267. ten Doesscate 146. Drüner 56.

E.

Egger 125.

Elschnig 138, 139, 142,

143, 186, 220. Engelking 197, 204,

206, 229, 273. Erb 56. Erdös 266. Erggelet 185, 238, 273. Eßner 57, 65, 66.

F.

Fej ér 263, 267. Fleischer 222. Friede 117. Friedenthal 128. Fuchs, A., 181, 196, 275.

G.

Gellhorn 202.

Giesler 244.

Gilbert 127, 146, 243,

246. Gjessing 79. Goldschmidt 127, 188,

232. Gonin 202. Goslich 38, 40. I Goulden 69.

genarbeiten.

v. Grosz 220, 264, 267 Grünberg 57. Grunert 250. Grüter 212. Guist 59, 255. Gulkowitsch 128.

H.

Hamburger, 130, 189,

216, 238. Hammer 364. Hanssen 35, 243, 244. Hartinger 57. Hauptvogel 139, 140. Heine 74, 155, 221. Henker 57, 60. Hertel 58. Heßberg 65, 67, 214,

$219,223,359,363$,

$367,369,37^{\circ}$. Heuser 66. van Heuven 144. Heyder 264, 267. Hiroishi 129. Hirschberg 274. van der Hoeve 144,

145, 195, 211. v. Hofe 64. Hoffmann, W. 21, 230. Honti 262. v. Horváth 56, 265,

267. Huber 262, 265.

I.

Igersheimer 180, 213,

219. v. Imre 189, 199, 260,

2. 263

267. v. Incze 261

267.

Jaensch 197. Jeß 237. 